\title{
IntelliGO: a new vector-based semantic similarity measure including annotation origin
}

\author{
Sidahmed Benabderrahmane ${ }^{1 *}$, Malika Smail-Tabbone ${ }^{1}$, Olivier Poch², Amedeo Napoli', \\ Marie-Dominique Devignes ${ }^{1}$
}

\begin{abstract}
Background: The Gene Ontology (GO) is a well known controlled vocabulary describing the biological process, molecular function and cellular component aspects of gene annotation. It has become a widely used knowledge source in bioinformatics for annotating genes and measuring their semantic similarity. These measures generally involve the GO graph structure, the information content of GO aspects, or a combination of both. However, only a few of the semantic similarity measures described so far can handle GO annotations differently according to their origin (i.e. their evidence codes).

Results: We present here a new semantic similarity measure called IntelliGO which integrates several complementary properties in a novel vector space model. The coefficients associated with each GO term that annotates a given gene or protein include its information content as well as a customized value for each type of GO evidence code. The generalized cosine similarity measure, used for calculating the dot product between two vectors, has been rigorously adapted to the context of the GO graph. The IntelliGO similarity measure is tested on two benchmark datasets consisting of KEGG pathways and Pfam domains grouped as clans, considering the GO biological process and molecular function terms, respectively, for a total of 683 yeast and human genes and involving more than 67,900 pair-wise comparisons. The ability of the IntelliGO similarity measure to express the biological cohesion of sets of genes compares favourably to four existing similarity measures. For inter-set comparison, it consistently discriminates between distinct sets of genes. Furthermore, the IntelliGO similarity measure allows the influence of weights assigned to evidence codes to be checked. Finally, the results obtained with a complementary reference technique give intermediate but correct correlation values with the sequence similarity, Pfam, and Enzyme classifications when compared to previously published measures.
\end{abstract}

Conclusions: The IntelliGO similarity measure provides a customizable and comprehensive method for quantifying gene similarity based on $\mathrm{GO}$ annotations. It also displays a robust set-discriminating power which suggests it will be useful for functional clustering.

Availability: An on-line version of the IntelliGO similarity measure is available at: http://bioinfo.loria.fr/Members/ benabdsi/intelligo_project/

\section{Background}

\subsection{Gene annotation}

The Gene Ontology (GO) has become one of the most important and useful resources in bioinformatics [1]. This ontology of about 30,000 terms is organized as a controlled vocabulary describing the biological process (BP), molecular function (MF), and cellular component

\footnotetext{
* Correspondence: benabdsi@loria.fr

'LORIA (CNRS, INRIA, Nancy-Université), Équipe Orpailleur, Bâtiment B,

Campus scientifique, 54506 Vandoeuvre-lès-Nancy Cedex, France

Full list of author information is available at the end of the article
}

(CC) aspects of gene annotation, also called GO aspects [2]. The GO vocabulary is structured as a rooted Directed Acyclic Graph (rDAG) in which GO terms are the nodes connected by different hierarchical relations (mostly is_a and part_of relations). The is-a relation describes the fact that a given child term is a specialization of a parent term, while the part-of relation denotes the fact that a child term is a component of a parent term. Another GO relation regulates expresses the fact that one process directly affects the manifestation of another process or quality [3]. However, this relation is

\section{Ciomed Central}


not considered in most studies dealing with semantic similarity measures. By definition, each rDAG has a unique root node, relationships between nodes are oriented, and there are no cycles, i.e. no path starts and ends at the same node.

The GO Consortium regularly updates a GO Annotation (GOA) Database [4] in which appropriate GO terms are assigned to genes or gene products from public databases. GO annotations are widely used for data mining in several bioinformatics domains, including gene functional analysis of DNA microarrays data [5], gene clustering [6-8], and semantic gene similarity [9].

It is worth noting that each GO annotation is summarized by an evidence code (EC) which traces the procedure that was used to assign a specific GO term to a given gene [10]. Out of all available ECs, only the Inferred from Electronic Annotation (IEA) code is not assigned by a curator. Manually assigned ECs fall into four general categories (see Section 2.4.3 and Table 1): author statement, experimental analysis, computational analysis, and curatorial statements. The author statement (Auth) means that the annotation either cites a published reference as the source of information (TAS for Traceable Author Statement) or it does not cite a published reference (NAS for Non traceable Author Statement). An experimental (Exp) annotation means that the annotation is based on a laboratory experiment. There are five ECs which correspond to various specific types of experimental evidence (IDA, IPI, IMP, IGI, and IEP; see Table 1 for details), plus one non specific parent code which is simply denoted as Exp. The use of an Exp EC annotation is always accompanied by the citation of a published reference. A Comp means that the annotation is based on computational analysis performed under the supervision of a human annotator. There are six types of Comp EC which correspond to various specific computational analyses (ISS, RCA, ISA, ISO, ISM and IGC; see Table 1 for details). The curatorial statement (Cur) includes the IC (Inferred by Curator) code which is used when an annotation is not supported by any direct evidence but can be reasonably inferred by a curator from other GO annotations for which evidence is available. For example, if a gene product has been annotated as a transcription factor on some experimental basis, the curator may add an IC annotation to the cellular component term nucleus. The ND (No biological Data available) code also belongs to the Cur category and means that a curator could not find any biological information. In practice, annotators are asked to follow a detailed decision tree in order to qualify each annotation with the proper EC [11]. Ultimately, a reference can describe multiple methods, each of which provides evidence to assign a certain GO term to a particular gene product. It is therefore common to see multiple gene annotations with identical GO identifiers but different ECs.

The statistical distribution of gene annotations with respect to the various ECs is shown in Figure 1 for human and yeast BP and MF aspects. This figure shows that IEA annotations are clearly dominant in both species and for all GO aspects, but that some codes are not represented at all (e.g. ISM, IGC).

However, the ratio between non-IEA and IEA annotations is different in yeast and human. It is about 2.0 and 0.8 for the yeast BP and MF annotations compared to about 0.8 and 0.6 for the corresponding human annotations, respectively. This observation reflects a higher contribution of non-IEA annotation in yeast and is somewhat expected because of the smaller size of yeast genome and because more experiments have been carried out on yeast. In summary, GO ECs add high value to gene annotations because they trace annotation origins. However, apart for the G-SESAME and SimGIC measures which select $\mathrm{GO}$ annotations on the basis of ECs [12], only a few of the gene similarity measures described so far can handle GO annotations differently according to their ECs [9], [13]. Hence, one objective of this paper is to introduce a new semantic similarity measure which takes into account GO annotations and their associated ECs.

Table 1 EC weight lists assigned to the 16 GO ECs considered in this study

\begin{tabular}{|c|c|c|c|c|c|c|c|c|c|c|c|c|c|c|c|c|c|}
\hline & \multicolumn{2}{|c|}{ Auth } & \multicolumn{8}{|c|}{ Exp } & \multicolumn{4}{|c|}{ Comp } & \multicolumn{2}{|c|}{ Cur } & \multirow{2}{*}{$\begin{array}{c}\text { Auto } \\
\text { IEA }\end{array}$} \\
\hline EC & TAS & NAS & EXP & IDA & IPI & IMP & IGI & IEP & ISS & RCA & ISA & ISO & ISM & IGC & IC & ND & \\
\hline List1 & & & & & & & & & 1 & & & & & & & & \\
\hline List2 & 1 & 0.5 & 0.8 & 0.8 & 0.8 & 0.8 & 0.8 & 0.8 & 0.6 & 0.6 & 0.6 & 0.6 & 0.6 & 0.6 & 0.5 & 0 & 0.4 \\
\hline List3 & 1 & 0.5 & 0.8 & 0.8 & 0.8 & 0.8 & 0.8 & 0.8 & 0.6 & 0.6 & 0.6 & 0.6 & 0.6 & 0.6 & 0.5 & 0 & 0 \\
\hline List4 & & & & & & & & & & & & & & & & & 1 \\
\hline
\end{tabular}

Table 1: The various weights assigned to the ECs are listed in the following lines as EC weight lists 1 to 4 . TAS: Traceable Author Statement; NAS: Non-traceable Author Statement; EXP: Inferred from Experiment; IDA: Inferred from Direct Assay; IPI: Inferred from Physical Interaction; IMP: Inferred from Mutant Phenotype; IGI: Inferred from Genetic Interaction; IEP: Inferred from Expression Pattern; ISS: Inferred from Sequence Similarity; RCA: Inferred from Reviewed Computational Analysis; ISA: Inferred from Sequence Alignment; ISO: Inferred from Sequence Orthology; ISM: Inferred from Sequence Model; IGC: Inferred from Genomic Context; IC: Inferred from Curator; IEA: Inferred from Electronic Annotation; ND: No biological Data available. The EC categories are indicated in the first line of the table. Auth: Author statement; Exp: Experimental; Comp: Computational Analysis; Cur: Curator statement; Auto: Automatically assigned. 


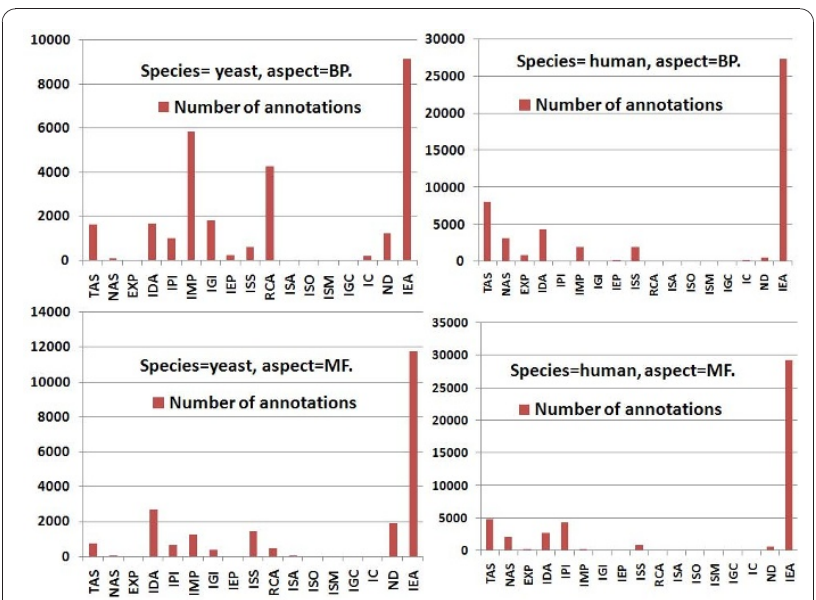

Figure 1 Distribution of EC (evidence codes) in yeast and human gene annotations according to BP and MF aspects. The number of annotations assigned to a gene with a given EC is represented for each EC. Note that some genes can be annotated twice with the same term but with a different EC. The cumulative numbers of all non-IEA annotations are 18,496 and 9,564 for the yeast BP and MF annotations, respectively, and 21,462 and 16,243 for the human BP and MF annotations, respectively. Statistics are derived from the NCBI annotation file, version June 2009.

\subsection{Semantic similarity measure}

\subsubsection{The notion of semantic similarity measure}

Using the general notion of similarity to identify objects which share common attributes or characteristics appears in many contexts such as word sense disambiguation, spelling correction, and information retrieval [14,15]. Similarity methods based on this notion are often called featural approaches because they assume that items are represented by lists of features which describe their properties. Thus, a similarity comparison involves comparing the feature lists that represent the items [16].

A similarity measure is referred to as semantic if it can handle the relationships that exist between the features of the items being compared. Comparing documents described by terms from a thesaurus or an ontology typically involves measuring semantic similarity [17]. Authors such as Resnik [18] or Jiang and Conrath [19] are considered as pioneers in ontology-based semantic similarity measures thanks to their long investigations in general English linguistics [20]. A general framework for comparing semantic similarity measures in a subsumption hierarchy has been proposed by Blanchard et al. [15]. For these authors, tree-based similarities fall into two large categories, namely those which only depend on the hierarchical relationships between the terms [21] and those which incorporate additional statistics such as term frequency in a corpus [22].

In the biological domain, the term functional similarity was introduced to describe the similarity between genes or gene products as measured by the similarity between their GO functional annotation terms. Biologists often need to establish functional similarities between genes. For example, in gene expression studies, correlations have been demonstrated between gene expression and GO semantic similarities [23,24]. Because GO terms are organized in a rDAG, the functional similarity between genes can be calculated using a semantic similarity measure. In a recent review, Pesquita et al. define a semantic similarity measure as a function that, given two individual ontology terms or two sets of terms annotating two biological entities, returns a numerical value reflecting the closeness in meaning between them [9]. These authors distinguish the comparison between two ontology terms from the comparison between two sets of ontology terms.

\subsubsection{Comparison between two terms}

Concerning the comparison between individual ontology terms, the two types of approaches reviewed by Pesquita et al. [9] are similar to those proposed by Blanchard et al. [15], namely the edge-based measures which rely on counting edges in the graph, and node-based measures which exploit information contained in the considered term, its descendants and its parents.

In most edge-based measures, the Shortest Path-Length (SPL) is used as a distance measure between two terms in a graph. This indicator was used by Rada et al. [25] on $\mathrm{MeSH}$ (Medical Subject Headings) terms and by AlMubaid et al. [26] on GO terms. However, Pesquita et al. question whether SPL-based measures truly reflect the semantic closeness of two terms. Indeed these measures rely on two assumptions that are seldom true in biological ontologies, namely that nodes and edges are uniformly distributed, and that edges at the same level in a hierarchy correspond to the same semantic distance between terms. Node-based measures are probably the most cited semantic similarity measures. These mainly rely on the information content (IC) of the two terms being compared and of their closest common ancestor $[18,22]$. The information content of a term is based on its frequency, or probability, of occurring in a corpus. Resnik uses the negative logarithm of the probability of a term to quantify its information content, $I C\left(c_{i}\right)=-\log$ $\left(p\left(c_{i}\right)\right)[18,27]$. Thus, a term with a high probability of occurring has a low IC. Conversely, very specific terms that are less frequent have a high IC. Intuitively, IC values increase as a function of depth in the hierarchy. Resnik's similarity measure between two terms consists of determining the IC of all common ancestors between two terms and selecting the maximal value, i.e. the IC of the most specific (i.e. lowest) common ancestor (LCA). In other words, if two terms share an ancestor with a high information content, they are considered to be semantically very similar. Since the maximum of this IC 
value can be greater than one, Lin introduced a normalization term into Resnik's measure yielding [22]:

$$
\operatorname{SIM}_{L i n}\left(c_{i}, c_{j}\right)=2 * \frac{\operatorname{IC}\left(\operatorname{LCA}\left(c_{i}, c_{j}\right)\right)}{\operatorname{IC}\left(c_{i}\right)+\operatorname{IC}\left(c_{j}\right)} .
$$

Recently, Schlicker et al. improved Lin's measure by using a correction factor based on the probability of occurrence of the $L C A$. Indeed, a general ancestor should not bring too high a contribution to term comparison [28]. A limitation of node-based measures is that they cannot explicitly take into account the distance separating terms from their common ancestor [9]. Hybrid methods also exist which combine edge-based and node-based methods, such as those developed by Wang et al. [29] and Othman et al. [30].

\subsubsection{Comparison between sets of terms}

Concerning the comparison between sets of terms, the approaches reviewed by Pesquita et al. fall into two broad categories: pairwise methods which simply combine the semantic similarities between all pairs of terms, and groupwise methods which consider a set of terms as a mathematical set, a vector, or a graph. The various pairwise methods differ in the strategies chosen to calculate the pairwise similarity between terms and in how pairwise similarities are combined. These methods have been thoroughly reviewed previously [9]. Hence we concentrate here on two representative examples that we chose for comparison purposes, namely the Lord measure which uses the node-based Resnik measure in the pairwise comparison step, and the Al-Mubaid measure which uses an edge-based measure. The study by Lord et al. in 2003 [2] provides the first description of a semantic similarity measure for GO terms. Semantic similarity between proteins is calculated as the average of all pairwise Resnik similarities between the corresponding GO annotations. In contrast, the measure defined by Al-Mubaid et al. [26], [31] considers the shortest path length (SPL) matrix between all pairs of GO terms that annotate two genes or gene products. It then calculates the average of all SPL values in the matrix, which represents the path length between two gene products. Finally, a transfer function is applied to the average SPL to convert it into a similarity value (see Methods). In group-wise methods, non semantic similarity measures co-exist with semantic ones. For example, the early Jaccard and Dice methods of counting the percentage of common terms between two sets are clearly non semantic [15]. However, in subsequent studies, various authors used sets of GO terms that have been extended with all term ancestors [32], [33].

Graph-based similarity measures are currently implemented in the Bioconductor GOstats package [34]. Each protein or gene can be associated with a graph which is induced by taking the most specific GO terms annotating the protein, and by finding all parents of those terms up to the root node. The union-intersection and longest shared path (SimUI) method can be used to calculate the between-graph similarity, for example. This method was tested by Guo et al. on human regulatory pathways [35]. Recently, the SimGIC method was introduced to improve the SimUI method by weighting terms with their information content [36].

Finally, vector-based similarity measures need to define an annotation Vector-Space Model (VSM) by analogy to the classical VSM described for document retrieval [37], [38], [39]. In the annotation VSM, each gene is represented by a vector $\vec{g}$ in a $k$-dimensional space constructed from basis vectors $\vec{e}_{i}$ which correspond to the $k$ annotation terms $[40,41]$. Thus, text documents and terms are replaced by gene and annotation terms, respectively, according to

$$
\vec{g}=\sum_{i} \alpha_{i} * \vec{e}_{i}
$$

where $\vec{e}_{i}$ is the $i$-th basis vector in the VSM annotation corresponding to the annotation term $t_{i}$, and where $\alpha_{i}$ is the coefficient of that term.

The DAVID tool, which was developed for functional characterization of gene clusters [6], uses this representation with binary coefficients which are set to 1 if a gene is annotated by a term and zero otherwise. Similarity is then calculated using "Kappa statistics" [42] which consider the significance of observed co-occurrences with respect to chance. However, this approach does not take into account the semantic similarity between functional annotation terms. In another study by Chabalier et al., the coefficients are defined as weights corresponding to the information content of each annotation term. The similarity between two genes is then computed using a cosine similarity measure. The semantic feature in Chabalier's method consists of a pre-filtering step which retains only those $\mathrm{GO}$ annotations at a certain level in the GO graph.

Ganesan et al. introduced a new vector-based semantic similarity measure in the domain of information retrieval [14]. When two annotation terms are different, this extended cosine measure allows the dot product between their corresponding vectors to be non-zero, thus expressing the semantic similarity that may exist between them. In other words, the components of the vector space are not mutually orthogonal. We decided to use this approach in the context of GO annotations. Hence the IntelliGO similarity measure defines a new vector-based representation of gene annotations with 
meaningful coefficients based on both information content and annotation origin. Vector comparison is based on the extended cosine measure and involves an edgebased similarity measure between each vector component.

\section{Results}

\subsection{The IntelliGO Vector Space Model to represent gene} annotations

\subsubsection{The IntelliGO weighting scheme}

The first originality of the IntelliGO VSM lies in its weighting scheme. The coefficients assigned to each vector component (GO term) are composed of two measures analogous to the $t f$-idf measures used for document retrieval [43]. On one hand, a weight $w\left(g, t_{i}\right)$ is assigned to the EC that traces the annotation origin and qualifies the importance of the association between a specific GO term $t_{i}$ and a given gene $g$. On the other hand, the Inverse Annotation Frequency (IAF) measure is defined for a given corpus of annotated genes as the ratio between the total number of genes $G_{T o t}$ and the number of genes $G_{t_{i}}$ annotated by the term $t_{i}$. The IAF value of term $t_{i}$ is calculated as

$$
\operatorname{IAF}\left(t_{i}\right)=\log \frac{G_{T o t}}{G_{t_{i}}} .
$$

This definition is clearly related to what was defined above as the information content of a GO term in an annotation corpus. It can be verified that GO terms which are frequently used to annotate genes in a corpus will display a low $I A F$ value, whereas $\mathrm{GO}$ terms that are rarely used will display a high $I A F$ which reflects their specificity and their potentially high contribution to vector comparison. In summary, the coefficient $\alpha_{i}$ is defined as

$$
\alpha_{i}=w\left(g, t_{i}\right) * \operatorname{IAF}\left(t_{i}\right) .
$$

\subsubsection{The IntelliGO generalized cosine similarity measure}

The second innovative feature of the IntelliGO VSM concerns the basis vectors themselves. In classical VSMs, the basis is orthonormal, i.e. the base vectors are normed and mutually orthogonal. This corresponds to the assumption that each dimension of the vector space (here each annotation term) is independent from the others. In the case of gene annotation, this assumption obviously conflicts with the fact that GO terms are interrelated in the GO rDAG structure. Therefore, in the IntelliGO VSM, basis vectors are not considered as orthogonal to each other within a given $\mathrm{GO}$ aspect (BP, MF, or CC).
A similar situation has been handled by Ganesan $e t$ al. [14] in the context of document retrieval using a treehierarchy of indexating terms. Given two annotation terms, $t_{i}$ and $t_{j}$, represented by their vectors, $\vec{e}_{i}$ and $\vec{e}_{j}$, respectively, the Generalized Cosine-Similarity Measure (GCSM) defines the dot product between these two base vectors as

$$
\vec{e}_{i} * \vec{e}_{j}=2 * \frac{\operatorname{Depth}\left(L C A\left(t_{i}, t_{j}\right)\right)}{\operatorname{Depth}\left(t_{i}\right)+\operatorname{Depth}\left(t_{j}\right)} .
$$

The GCSM measure has been applied successfully by Blott $e t$ al. to a corpus of publications indexed using $\mathrm{MeSH}$ terms [43]. However applying the GCSM to the GO rDAG is not trivial. As mentioned above, in an rDAG there exist more than one path from one term to the Root. This has two consequences for the GCSM formula (5). Firstly, there may exist more than one LCA for two terms. Secondly, the depth value of a term is not unique but depends on the path which is followed up to the rDAG root. We therefore adapted the GCSM formula to rDAGs in a formal approach inspired by Couto et al. [44].

The GO controlled vocabulary can be defined as a triplet $\gamma=(T, \Xi, R)$, where $T$ is the set of annotation terms, $\Xi$ is the set of the two main hierarchical relations that may hold between terms, i.e. $\Xi=\{$ is-a, part-of $\}$. The third element $R$ contains a set of triples $\tau=\left(t, t^{\prime}\right.$, $\xi)$, where $t, t^{\prime} \in T, \xi \in \Xi$ and $t \xi t^{\prime}$. Note that $\xi$ is an oriented child-parent relation and that $\forall \tau \in R$, the relation $\xi$ between $t$ and $t^{\prime}$ is either is- $a$ or part-of. In the $\gamma$ vocabulary, the Root term represents the top-level node of the GO rDAG. Indeed, Root is the direct parent of three nodes, BiologicalProcess, CellularComponent, and MolecularFunction. These are also called aspect-specific roots. The Root node does not have any parents, and hence the collection $R$ does not contain any triple in which $t=$ Root. All GO terms in $T$ are related to the root node through their aspect-specific root. Let Parents be a function that returns the set of direct parents of a given term $t$ :

$$
\begin{gathered}
\text { Parents : } T \rightarrow \mathcal{P}(T), \\
\operatorname{Parents}(t)=\left\{t^{\prime} \in T \quad \mid \exists \xi \in \Xi, \exists \tau \in R, \quad \tau=\left(t, t^{\prime}, \xi\right)\right\},
\end{gathered}
$$

where $\mathcal{P}(T)$ refers to the set of all possible subsets of $T$. Note that Parents $($ Root $)=\varnothing$. The function Parents is used to define the RootPath function as the set of directed paths descending from the Root term to a given term $t$ : 


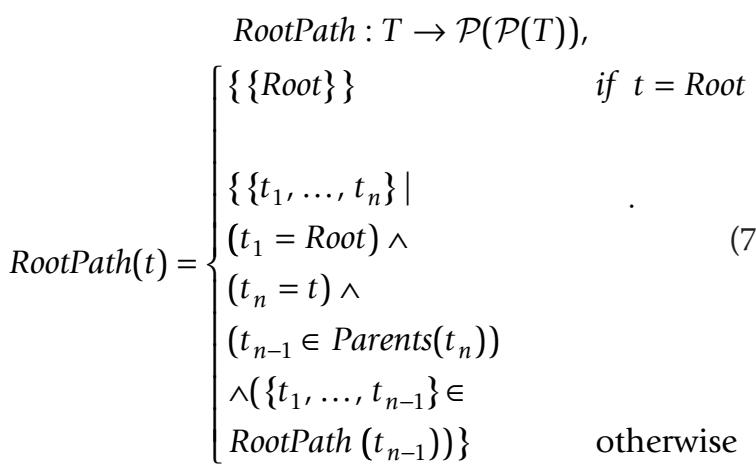

Thus, each path between the Root term and a term $t$ is a set of terms $\Phi \in \operatorname{RootPath}(t)$.

The length of a path separating a term $t$ from the Root term is defined as the number of edges connecting the nodes in the path, and is also called the Depth of term $t$. However, due to the multiplicity of paths in rDAG, there can be more than one depth value associated with a term. In the following, and by way of demonstration, we define $\operatorname{Depth}(t)$ as the function associating a term $t$ with its maximal depth:

$$
\operatorname{Depth}(t)=\operatorname{Max}_{i}\left(\left|\Phi_{i}\right|\right)-1 \quad \mid \Phi_{i} \in \operatorname{RootPath}(t) .
$$

Note that since RootPath $($ Root $)=\{\{$ Root $\}\}$, we have $\operatorname{Depth}(\operatorname{Root})=|\{\operatorname{Root}\}|-1=0$.

We then define the Ancestors function to identify an ancestor term of a given term $t$ as any element $\alpha$ of a path $\Phi \in \operatorname{RootPath}(t)$.

$$
\text { Ancestors : } T \rightarrow P(T)
$$

and

$$
\operatorname{Ancestors}(t)=\{\alpha \in T \mid \exists \Phi,(\Phi \in \operatorname{RootPath}(t)) \wedge(\alpha \in \Phi)\} .
$$

Thus, the common ancestors of two terms $t_{a}$ and $t_{b}$ can be defined as:

$$
\operatorname{CommonAnc}\left(t_{a}, t_{b}\right)=\operatorname{Ancestors}\left(t_{a}\right) \cap \operatorname{Ancestors}\left(t_{b}\right) .
$$

Let $L C A s e t\left(t_{a}, t_{b}\right)$ be the set of lowest common ancestors of terms $t_{a}, t_{b}$. The lowest common ancestors are at the maximal distance from the root node. In other words their depth is the maximum depth of all terms $\alpha$ $\in \operatorname{CommonAnc}\left(t_{a}, t_{b}\right)$. Note that this value is unique but it may correspond to more than one $L C A$ term:

$$
\begin{aligned}
& \text { LCAset }: T \mathrm{x} T \rightarrow \mathcal{P}(T), \\
& \qquad \begin{aligned}
\text { LCAset }\left(t_{a}, t_{b}\right)= & \left\{\alpha \in \operatorname{CommonAnc}\left(t_{a}, t_{b}\right) \mid\right. \\
& \operatorname{Depth}(\alpha)=\operatorname{Max}_{i}\left(\operatorname{Depth}\left(a_{i}\right)\right), \\
& \left.a_{i} \in \operatorname{CommonAnc}\left(t_{a}, t_{b}\right)\right\} .
\end{aligned}
\end{aligned}
$$

Having defined the LCAset, it is possible to define a subset of paths from the Root term to a given term $t$ that pass through one of the $L C A$ terms and subsequently ascend to the root node using the longest path between the LCA and the Root term. This notion is called ConstrainedRootPath, and can be calculated for any pair $(\mathrm{t}, \mathrm{s})$ with $s \in \operatorname{Ancestors}(t)$ :

$$
\begin{gathered}
\text { ConstrainedRootPath }(t, s)=\left\{\begin{array}{c}
\left\{\Phi_{i} \in \operatorname{RootPath}(t) \mid\right. \\
\left(s \in \Phi_{i}\right) \wedge \\
\left(\forall \Phi_{j},\left(\left(\Phi_{j} \in \operatorname{RootPath}(s)\right) \wedge\right.\right. \\
\left.\left.\left.\left(\Phi_{j} \subset \Phi_{i}\right)\right) \Rightarrow\left(\left|\Phi_{j}\right|=\operatorname{Depth}(s)+1\right)\right)\right\}
\end{array}\right.
\end{gathered}
$$

This leads to a precise definition of the path length $P L_{k}(t, s)$, for $s \in$ Ancestors $(t)$ and for a given path $\Phi_{k} \in$ ConstrainedRootPath $(t, s)$ as:

$$
P L_{k}\left(t_{i}, s\right)=\left|\Phi_{k}\right|-1-\operatorname{Depth}(s) .
$$

For a given $L C A \in L C A \operatorname{set}\left(t_{i}, t_{j}\right)$, we can now define the shortest path length (SPL) between two terms $t_{i}$ and $t_{j}$ passing through this lowest common ancestor as

$$
\begin{array}{r}
S P L\left(t_{i}, t_{j}, L C A\right)=\operatorname{Min}_{k}\left(P L_{k}\left(t_{i}, L C A\right)\right)+ \\
\operatorname{Min}_{h}\left(P L_{h}\left(t_{j}, L C A\right)\right) .
\end{array}
$$

The minimal SPL between terms $t_{i}$ and $t_{j}$ considering all their possible $L C A$ s is thus given by

$$
\begin{array}{r}
\operatorname{MinSPL}\left(t_{i}, t_{j}\right)=\operatorname{Min}_{l}\left(\operatorname{SPL}\left(t_{i}, t_{j}, L C A_{l}\right)\right) \mid \\
\operatorname{LCA}_{l} \in \operatorname{LCAset}\left(t_{i}, t_{j}\right) .
\end{array}
$$

Returning to the GCSM formula (5), we now relate Depth $\left(t_{i}\right)+\operatorname{Depth}\left(t_{j}\right)$ in the denominator of the expression with $\operatorname{MinSPL}\left(t_{i}, t_{j}\right)$ and $\operatorname{Depth}(L C A)$. Note that from (8) we have

$\operatorname{Depth}\left(t_{i}\right)=\operatorname{Max}_{k}\left(\left|\Phi_{k}\right|-1\right)$, with $\Phi_{k} \in \operatorname{RootPath}\left(t_{i}\right)$. From (13) we have

$P L_{k}\left(t_{i}, L C A\right)=\left|\Phi_{k}\right|-1-\operatorname{Depth}(L C A)$ with $\Phi_{k} \in$ ConstrainedRootPath $\left(t_{i}, L C A\right)$ and ConstrainedRootPath $\left(t_{i}\right.$, $L C A) \subset \operatorname{RootPath}(t)$. Given any $L C A \in L C A s e t\left(t_{i}, t_{j}\right)$, it is then easy to demonstrate that

$$
\operatorname{Depth}\left(t_{i}\right) \geq \operatorname{Min}_{k}\left(P L_{k}\left(t_{i}, L C A\right)\right)+\operatorname{Depth}(L C A) .
$$

Similarly,

$$
\operatorname{Depth}\left(t_{j}\right) \geq \operatorname{Min}_{h}\left(P L_{h}\left(t_{j}, L C A\right)\right)+\operatorname{Depth}(L C A) .
$$

Thus,

$$
\begin{array}{r}
\operatorname{Depth}\left(t_{i}\right)+\operatorname{Depth}\left(t_{j}\right) \geq \\
\operatorname{SPL}\left(t_{i}, t_{j}, L C A\right)+2 * \operatorname{Depth}(L C A) \geq \\
\operatorname{MinSPL}\left(t_{i}, t_{j}\right)+2 * \operatorname{Depth}(L C A) .
\end{array}
$$


In the case of a tree, this inequality becomes an equality.

The semantic similarity between two terms is assumed to be inversely proportional to the length of the path separating the two terms across their LCA. When we adapt the GCSM measure in (5) by replacing in the denominator the sum $\operatorname{Depth}\left(t_{i}\right)+\operatorname{Depth}\left(t_{j}\right)$ by the smaller sum $\operatorname{MinSPL}\left(t_{i}, t_{j}\right)+2$ "Depth(LCA), we ensure that the dot product between two base vectors will be maximized. With this adaptation, the IntelliGO dot product between two base vectors corresponding to two GO terms $t_{i}$ and $t_{j}$ is defined as

$$
\vec{e}_{i} * \vec{e}_{j}=\frac{2 * \operatorname{Depth}(L C A)}{\operatorname{MinSPL}\left(t_{i}, t_{j}\right)+2 * \operatorname{Depth}(L C A)} .
$$

One can verify that with this definition, the dot product takes values in the interval $0[1]$. We observe that for $i=j, \quad \vec{e}_{i} * \vec{e}_{i}=1$, since $\operatorname{MinSPL}\left(t_{i}, t_{j}\right)=0$. Moreover, when two terms are only related through the root of the rDAG, we have $\vec{e}_{i} * \vec{e}_{i}=0$ because Depth(Root) $=0$. In any other case, the value of the dot product represents a non zero edge-based similarity between terms. Note that this value clearly depends on the rDAG structure of the GO graph.

\subsection{The IntelliGO semantic similarity measure}

In summary, the IntelliGO semantic similarity measure between two genes $g$ and $h$ represented by their vectors $\vec{g}$ and $\vec{h}$, respectively, is given by the following cosine formula:

$$
\operatorname{SIM}_{\text {IntelliGO }}(g, h)=\frac{\vec{g} * \vec{h}}{\sqrt{\vec{g} * \vec{g}} \sqrt{\vec{h} * \vec{h}}},
$$

where:

- $\vec{g}=\sum_{i} \alpha_{i} * \vec{e}_{i}$ : the vectorial representation of the gene $g$ in the IntelliGO VSM.

- $\vec{h}=\sum_{j} \beta_{j}{ }^{*} \vec{e}_{j}$ : the vectorial representation of the gene $h$ in the IntelliGO VSM.

- $\alpha_{i}=w\left(g, t_{i}\right)^{*} \operatorname{IAF}\left(t_{i}\right)$ the coefficient of term $t_{i}$ for gene $g$, where $\mathrm{w}\left(g, t_{i}\right)$ represents the weight assigned to the evidence code between $t_{i}$ and $g$, and $\operatorname{IAF}\left(t_{i}\right)$ is the inverse annotation frequency of the term $t_{i}$.

- $\beta_{j}=w\left(h, t_{j}\right)^{*} \operatorname{IAF}\left(t_{j}\right)$ the coefficient of term $t_{j}$ for gene $h$.

- $\vec{g} * \vec{h}=\sum_{i, j} \alpha_{i} * \beta_{j} * \vec{e}_{i} * \vec{e}_{j}$ : the dot product between the two gene vectors.
- $\vec{e}_{i} * \vec{e}_{j}=\frac{2 * \operatorname{Depth}(L C A)}{\operatorname{MinSPL}\left(t_{i}, t_{j}\right)+2 * \operatorname{Depth}(L C A)}:$ the dot

product between $\vec{e}_{i}$ and $\vec{e}_{j}\left(\vec{e}_{i} * \vec{e}_{j} \neq 0\right.$ if the corresponding terms $t_{i}$ and $t_{j}$ share common ancestors other than the rDAG root).

\subsection{The IntelliGO Algorithm}

The IntelliGO algorithm was designed to calculate the similarity measure between two genes, taking as input their identifiers in the NCBI GENE database, and as parameters a GO aspect (BP, MF, CC), a particular species, and a list of weights associated with GO ECs. The output is the IntelliGO similarity value between the two genes. In order to calculate this efficiently, we first extract from the NCBI annotation file [45] the list of all non redundant GO terms and the list of associated genes they annotate, whatever their evidence codes. The $I A F$ values are then calculated and stored in the SpeciesIAF file. We then construct all possible pairs of GO terms and query the AMIGO database [46] to recover their $L C A$, Depth $(L C A)$ and SPL values. Each dot product between two vectors representing two GO terms can thus be pre-calculated and stored in the DotProduct file.

The first step of the IntelliGO algorithm consists of filtering the NCBI file with the user's parameters (GO aspect, species and list of weights assigned to ECs) to produce a CuratedAnnotation file from which all genes of species and GO aspects other than those selected are removed. If a gene is annotated several times by the same GO term with different ECs, the program retains the EC having the greatest weight in the list of EC weights given as parameter. Then, for two input NCBI gene identifiers, the IntelliGO function (i) retrieves from the CuratedAnnotation file the list of GO terms annotating the two genes and their associated ECs, (ii) calculates from the SpeciesIAF file and the list of EC weights, all the coefficients of the two gene representations in the IntelliGO VSM, (iii) constructs the pairs of terms required to calculate the similarity value between the two vectors, (iv) assigns from the DotProduct file the corresponding value to each dot product, and (v) finally calculates the IntelliGO similarity value according to (20).

\subsection{Testing the IntelliGO semantic similarity measure 2.4.1 Benchmarking datasets and testing protocol}

We evaluated our method using two different benchmarks depending on the GO aspect. For the KEGG benchmark, we selected a representative set of 13 yeast and 13 human diverse KEGG pathways [47] which 
contain a reasonable number of genes (between 10 and $30)$. The selected pathways are listed in Table 2. The genes in these pathways were retrieved from KEGG using the DBGET database retrieval system [48]. Assuming that genes which belong to the same pathway are often related to a similar biological process, the similarity values calculated for this dataset should be related to the BP GO aspect.

For the Pfam benchmark, we selected a set of clans (groups of highly related Pfam entries) from the Sanger Pfam database [49]. In order to maximize diversity in the benchmarking dataset, yeast and human sequences were retrieved from the 10 different Pfam clans listed in Table 3. For each selected Pfam clan, we used all the associated Pfam entry identifiers to query the Uniprot database [50] and retrieve the corresponding human and yeast gene identifiers. Assuming that genes which share common domains in a Pfam clan often have a similar molecular function, the similarity values calculated for this second dataset should be related to the MF GO aspect.

For each set of genes, an intra-set average gene similarity was calculated as the average of all pairwise similarity values within a set of genes. In contrast, an interset average gene similarity was also calculated between two sets $S_{a}$ and $S_{b}$ as the average of all similarity values calculated for pairs of genes from each of the two sets $S_{a}$ and $S_{b}$. A discriminating power can then be defined according to the ratio of the intra-set and inter-set average gene similarities (see Methods). We compared the values obtained with the IntelliGO similarity measure with the four other representative similarity measures

Table 2 List of yeast and human pathways used in this study

\begin{tabular}{|c|c|c|c|c|c|c|c|}
\hline KEGG & KEGG & Yeast & Name & $\begin{array}{l}\mathrm{Nb} \\
\text { genes }\end{array}$ & Human & Name & $\begin{array}{c}\mathrm{Nb} \\
\text { genes }\end{array}$ \\
\hline Category & Subcategory & Pathway & & & Pathway & & \\
\hline \multirow[t]{8}{*}{01100 Metabolism } & $\begin{array}{l}01101 \text { Carbohydrate } \\
\text { Metabolism }\end{array}$ & sce00562 & $\begin{array}{l}\text { Inositol phosphate } \\
\text { metabolism }\end{array}$ & 15 & hsa00040 & $\begin{array}{l}\text { Pentose and glucuronate } \\
\text { interconversions }\end{array}$ & 26 \\
\hline & $\begin{array}{l}01102 \text { Energy } \\
\text { Metabolism }\end{array}$ & sce00920 & Sulfur metabolism & 13 & hsa00920 & Sulfur metabolism & 13 \\
\hline & 01103 Lipid Metabolism & sce00600 & $\begin{array}{l}\text { Sphingolipid } \\
\text { metabolism }\end{array}$ & 13 & hsa00140 & $\begin{array}{l}\text { C21-Steroid homone } \\
\text { metabolism }\end{array}$ & 17 \\
\hline & 01105 Amino Acid & sce00300 & Lysine biosynthesis & 13 & hsa00290 & $\begin{array}{c}\text { Valine, leucine and isoleucine } \\
\text { biosynthesis }\end{array}$ & 11 \\
\hline & Metabolism & sce00410 & Alanine biosynthesis & 8 & & & \\
\hline & $\begin{array}{c}01107 \text { Glycan } \\
\text { Biosynthesis and } \\
\text { Metabolism }\end{array}$ & sce00514 & $\begin{array}{l}\text { O-Mannosyl glycan } \\
\text { biosynthesis }\end{array}$ & 13 & hsa00563 & $\begin{array}{l}\text { Glycosylphosphatidylinositol } \\
\text { (GPI)-anchor biosynthesis }\end{array}$ & 23 \\
\hline & $\begin{array}{l}01109 \text { Metabolism of } \\
\text { Cofactors and Vitamins }\end{array}$ & sce00670 & $\begin{array}{l}\text { One carbon pool by } \\
\text { folate }\end{array}$ & 14 & hsa00670 & One carbon pool by folate & 16 \\
\hline & $\begin{array}{l}01110 \text { Biosynthesis of } \\
\text { Secondary Metabolites }\end{array}$ & sce00903 & $\begin{array}{l}\text { Limonene and pinene } \\
\text { degradation }\end{array}$ & 7 & hsa00232 & Caffeine metabolism & 7 \\
\hline \multirow[t]{5}{*}{$\begin{array}{c}01120 \text { Genetic } \\
\text { Information Processing }\end{array}$} & 01121 Transcription & sce03022 & $\begin{array}{l}\text { Basal transcription } \\
\text { factors }\end{array}$ & 24 & hsa03022 & Basal transcription factors & 38 \\
\hline & & & & & hsa03020 & RNA polymerase & 29 \\
\hline & $\begin{array}{l}01123 \text { Folding, Sorting } \\
\text { and Degradation }\end{array}$ & sce04130 & $\begin{array}{l}\text { SNARE interactionst in } \\
\text { vesicular Transport }\end{array}$ & 23 & hsa04130 & $\begin{array}{l}\text { SNARE interactions in } \\
\text { vesicular transport }\end{array}$ & 38 \\
\hline & $\begin{array}{l}01124 \text { Replication and } \\
\text { Repair }\end{array}$ & sce03450 & $\begin{array}{l}\text { Non-homologous } \\
\text { end-joining }\end{array}$ & 10 & hsa03450 & $\begin{array}{l}\text { Non-homologous end- } \\
\text { joining }\end{array}$ & 14 \\
\hline & & & & & hsa03430 & Mismatch repair & 23 \\
\hline $\begin{array}{l}01130 \text { Environmental } \\
\text { Information Processing }\end{array}$ & $\begin{array}{l}01132 \text { Signal } \\
\text { Transduction }\end{array}$ & sce04070 & $\begin{array}{l}\text { Phosphatidylinositol } \\
\text { signaling system }\end{array}$ & 15 & & & \\
\hline 01140 Cellular Processes & $\begin{array}{l}01151 \text { Transport and } \\
\text { Catabolism }\end{array}$ & sce 04140 & $\begin{array}{l}\text { Regulation of } \\
\text { autophagy }\end{array}$ & 17 & & & \\
\hline 01160 Human Diseases & $\begin{array}{l}01164 \text { Metabolic } \\
\text { Disorders }\end{array}$ & & & & hsa04950 & $\begin{array}{l}\text { Maturity onset diabetes of } \\
\text { the young }\end{array}$ & 25 \\
\hline Total genes number & & & & 185 & & & 280 \\
\hline Non-IEA:IEA ratio & & & & $\begin{array}{c}572: 435 \\
(1.3)\end{array}$ & & & $\begin{array}{l}560: 620 \\
(0.9)\end{array}$ \\
\hline
\end{tabular}

Table 2: The KEGG categories and subcategories are indicated for each pathway as well as its name and the number of genes it contains (KEGG version Dec 2009). The non-IEA:IEA ratio refers to Biological Process GO annotation of the complete set of genes for each species. 
Table 3 List of yeast and human genes and Pfam clans used this study

\begin{tabular}{|c|c|c|c|c|c|}
\hline Pfams clan accession (yeast) & $\mathrm{Nb}$ genes & Pfams clan name & Pfams clan accession (human) & $\mathrm{Nb}$ genes & Pfams clan name \\
\hline CL0328.1 & 15 & 2heme_cytochrom & CL0099.10 & 18 & ALDH-like \\
\hline CL0059.12 & 13 & 6_Hairpin & CL0106.10 & 8 & 6PGD_C \\
\hline CL0092.9 & 8 & ADF & CL0417.1 & 9 & BIR-like \\
\hline CL0099.10 & 11 & ALDH-like & CL0165.8 & 5 & Cache \\
\hline CL0179.11 & 11 & ATP-grasp & CL0149.9 & 7 & CoA-acyltrans \\
\hline CL0255.6 & 7 & ATP_synthase & CL0085.11 & 12 & FAD_DHS \\
\hline CL0378.1 & 10 & Ac-CoA-synth & CL0076.9 & 18 & FAD_Lum_binding \\
\hline CL0257.6 & 18 & Acetyltrans-like & CL0289.3 & 6 & $\mathrm{FBD}$ \\
\hline CL0034.12 & 11 & Amidohydrolase & CL0119.10 & 7 & Flavokinase \\
\hline CL0135.8 & 14 & Arrestin_N-like & CL0042.9 & 10 & Flavoprotein \\
\hline Total genes number & 118 & & & 100 & \\
\hline Non-IEA:IEA ratio & $121: 366(0.3)$ & & & $144: 309(0.46)$ & \\
\hline
\end{tabular}

Table 3: Clans are indicated by their accession identifier in the Sanger Pfam database (October 2009 release) and by the number of genes retrieved either in yeast (left part) or in human (right part). Each clan contains several Pfam entries listed in the Pfam_C file at [57]. The non-IEA:IEA ratio refers to the Molecular Function GO annotation of the complete set of genes for each species.

described above, namely the Lord measure which is based on the Resnick term-term similarity, the AlMubaid measure which considers only the path length between GO terms [31], a standard vector-based cosine similarity measure, and the SimGIC measure which is one of the graph-based methods described above (see Section 1.2.3). For each dataset, we evaluated our measure firstly by comparing the intra-set similarity values with those obtained with other measures, and then by studying the effect of varying the list of weights assigned to the ECs. We then compared the discriminating power of the IntelliGO similarity measure with three other measures. We also tested our measure on a reference dataset using a recently available on-line evaluation tool.

\subsubsection{Intra-set similarity}

We produced all intra-set similarities with the IntelliGO similarity measure using EC List1 (all weights set to 1.0, see Table 1). We also implemented and tested four other measures, namely Lord-normalized, Al-Mubaid, the classical weighted-cosine measure, and the SimGIC measure (see Methods) on the same sets of genes. The results obtained with the KEGG pathways using BP annotations are shown in Figure 2. For each KEGG pathway ( $\mathrm{x}$-axis), the intra-set similarity values are represented as histograms (y-axis). Similarity values vary from one pathway to another, reflecting variation in the coherence of gene annotations within pathways. Variations from one pathway to another are relatively uniform for all measures except the Lord measure. For example, intra-set similarity values of the sce00410 pathway are smaller than those of sce00300 for all measures except for the Lord measure. The same is observed between pathways hsa00920 and hsa00140.

A positive feature of the IntelliGO similarity measure is that unlike other measures, all intra-set values are greater than or equal to 0.5 . The relatively low values obtained with the weighted-cosine measure can be explained by the numerous null pairwise values generated by this method. This is because this measure assumes that the dimensions of the space vector are orthogonal to each other. Hence, whenever two genes lack a common annotation term their dot product is null, and so also is their similarity value. Indeed, null pairwise similarity values are observed in all pathways except for one in human and three in yeast (details not shown).

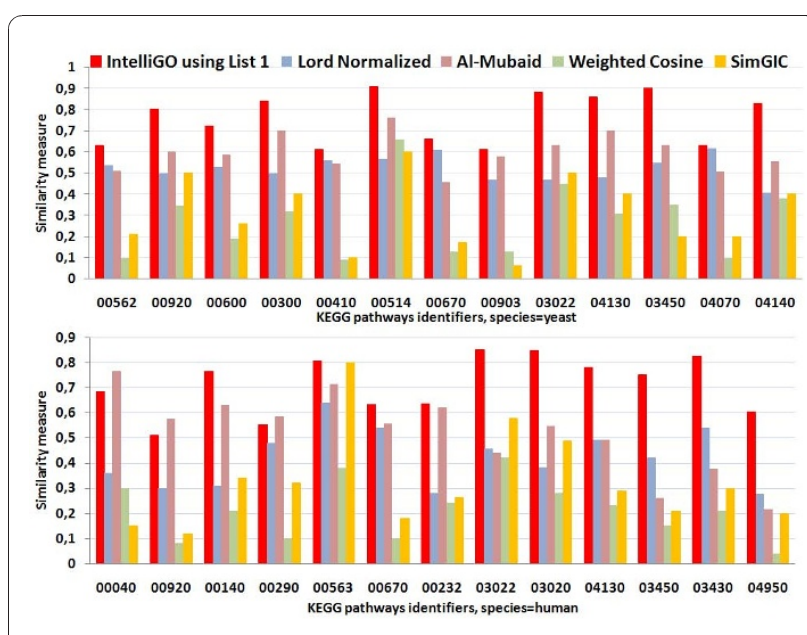

Figure 2 Intra-set similarities with the KEGG pathway dataset using BP annotations. The intra-set similarity is calculated as the mean of all pairwise gene similarities within a KEGG pathway, with the four measures compared in this study, namely, IntelliGO (using EC weight List1 ), Lord-normalized, Al-Mubaid, and Weighted-cosine. A set of thirteen pathways were selected from the KEGG Pathway database for yeast (top panel) and human (bottom panel) pathways. Only BP annotations are used here (see also Table 2). 
Very similar results were obtained with the Pfam benchmarking dataset which was analyzed on the basis of MF annotations (Figure 3). Here again, the IntelliGO similarity measure always provides intra-set similarity values greater than or equal to 0.5 , which is not the case for the other measures. As before, the weighted-cosine yields the lowest intra-set similarity values for the reason explained above. This inconvenience led us to skip this measure in later stages of the work. In summary, our comparison of intra-set similarity values for two benchmarking datasets demonstrates the robustness of the IntelliGO similarity measure and its ability to capture the internal coherence of gene annotation within predefined sets of genes.

\subsubsection{Influence of EC weight lists}

The second part of our evaluation is the study of the effect of varying the weights assigned to ECs in the IntelliGO similarity measure. As a first experiment, we used four lists of EC weights (see Table 1). In List1, all EC weights are equal to 1.0, which makes all ECs equivalent in their contribution to the similarity value. List1 was used above to compare the IntelliGO measure with the four other similarity measures (Figure 2 and 3) because these measures do not consider varying ECs weights in the calculation. In List2, the EC weights have been arbitrarily defined to represent the assumption that the Exp category of ECs is more reliable than the Comp category, and that the non-supervised IEA code is less reliable than Comp codes. List3 excludes IEA code in order to test the similarity measure when using only supervised annotations. Finally, List4 represents the

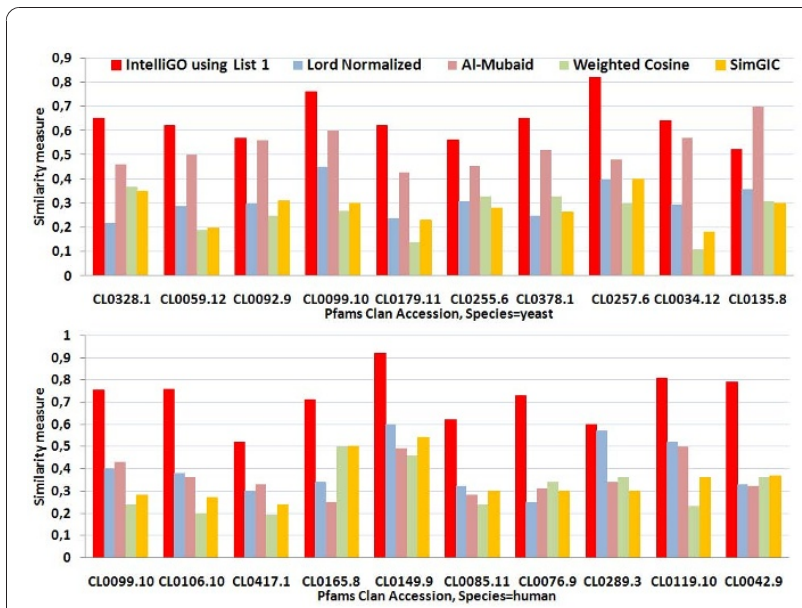

Figure 3 Intra-set similarities with the Pfam clan dataset using MF annotations. The intra-set similarity is calculated for all genes of a given species within a Pfam clan using MF annotations. Two collections of ten Pfam clans were selected from the Sanger Pfam database to retrieve yeast (top panel) and human (bottom panel) genes belonging to these clans (see also Table 3). opposite situation by retaining only the IEA code to test the contribution of IEA annotations.

These four lists were used to calculate IntelliGO intraset similarity values on the same datasets as before. For each dataset, the distribution of all pairwise similarity values used to calculate the intra-set averages is shown in Figure 4 with each weight list being shown as a histogram with a class interval of 0.2. On the left of each histogram a Missing Values bar (MV) shows the number of pairwise similarity values that cannot be calculated with List3 or List4 due to the complete absence of annotations for certain genes. As expected, since intra-set similarity values with the IntelliGO measure are greater than 0.5 , the highest numbers of pairwise values are found in the intervals $0.6-0.8$ and $0.8-1.0$ for all weight lists considered here. For List1 and List2, the distribution of values looks similar for all datasets. The effect of excluding the IEA code (List3 ) or considering it alone (List4) differs between the KEGG pathways and Pfam clans, i.e. between the BP and MF annotations. It also varies between the yeast and human datasets, reflecting the different ratios of IEA versus non-IEA annotations in these two species (see Figure 1 and Table 2 and 3). For the yeast KEGG pathways, the most striking variation is observed with List 4 which gives a marked decrease in the number of values in the 0.8-1.0 class interval, and a significant number of missing values.

This means that for this dataset, using only IEA BP annotations yields generally lower similarity values and

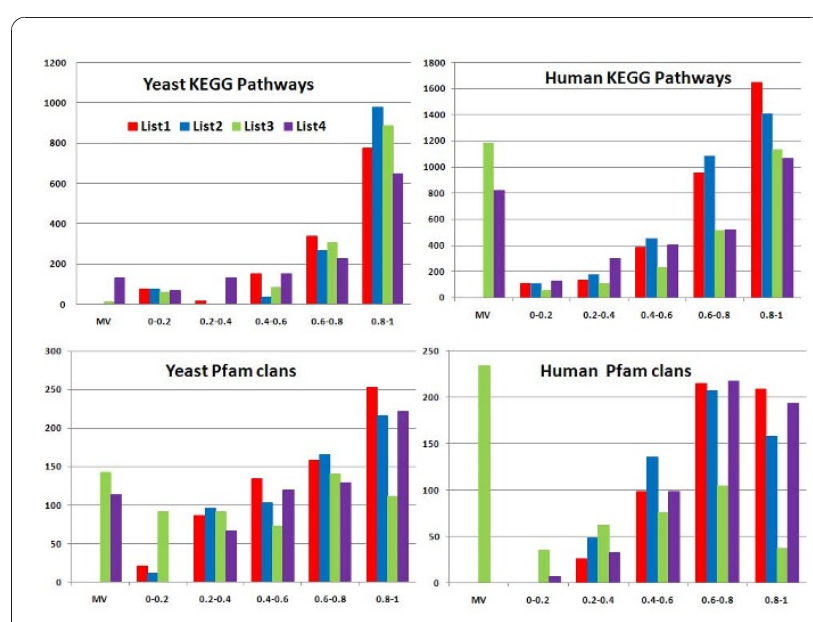

Figure 4 Influence of various EC weight lists on the distribution of pairwise similarity values obtained for intra-set similarity calculation. KEGG pathway datasets are handled with BP annotations, and Pfam clans with MF annotations. The MV bar is for Missing Values and represents the number of pairwise similarity values that cannot be calculated using List3 or List4 due to the missing annotations for certain genes. Pairwise similarity intervals are displayed on the $x$ axis of the histograms, while values on the $y$ axis represent the number of pairwise similarity values present in each interval. 
excludes from the calculation those genes without any IEA annotation (11 genes). This reflects the relatively high ratio (1.3) of non-IEA to IEA BP annotations in this dataset, and in yeast in general (2.0). A similar behavior is observed with the human KEGG pathways, not only with List4 but also with List3. The higher Missing Values bars in this dataset results from the high number of genes having either no IEA BP annotations (49 genes) or only IEA BP annotations (68 genes). This type of analysis shows that for such a dataset, IEA annotations are useful to capture intra-set pairwise similarity but they are not sufficient per se.

For the yeast and human Pfam clans, the distribution of values obtained with List3 is clearly shifted towards lower class intervals and Missing Values bars. This reflects the extent of the IEA MF annotations, and their important role in capturing intra-set similarity in these datasets (the ratios between non-IEA and IEA MF annotations are 0.3 and 0.46 for the yeast and human Pfam clan datasets, respectively). A total of 19 genes are annotated only with an IEA code in yeast, and 29 in human. Concerning List4, using only IEA MF annotations does not lead to large changes in the value distribution when compared to List 1 and List2. This suggests that these annotations are sufficient to capture intra-set pairwise similarity for such datasets. However, a significant number of missing values is observed in the yeast Pfam clan dataset, with 20 genes lacking any IEA MF annotations.

In summary, using customized weight lists for evidence codes in the IntelliGO measure is a useful way to highlight the contribution that certain types of annotations make to similarity values, as shown with the Pfam clan datasets and IEA MF annotations. However, this contribution clearly depends on the dataset and on the considered GO aspect. Other weight lists may be worth considering if there is special interest for certain ECs in certain datasets. In this study, we decided to continue our experiments with List2 since this weight list expresses the commonly shared view about the relative importance of ECs for gene annotation.

\subsubsection{Discriminating power}

The third step of our evaluation consisted of testing the Discriminating Power (DP) of the IntelliGO similarity measure and comparing it with three other measures (Lord-normalized, Al-Mubaid, and SimGIC). The calculation of a discriminating power is introduced here to evaluate the ability of a similarity measure to distinguish between two functionally different sets of genes. The DP values for these three measures for the two benchmarking datasets are plotted in Figure 5 and 6. For the KEGG pathways and BP annotations (Figure 5), the IntelliGO similarity measure produces $D P$ values greater than or equal to 1.3 for each tested pathway, with a maximum of 2.43 for the hsa04130 pathway. In contrast,

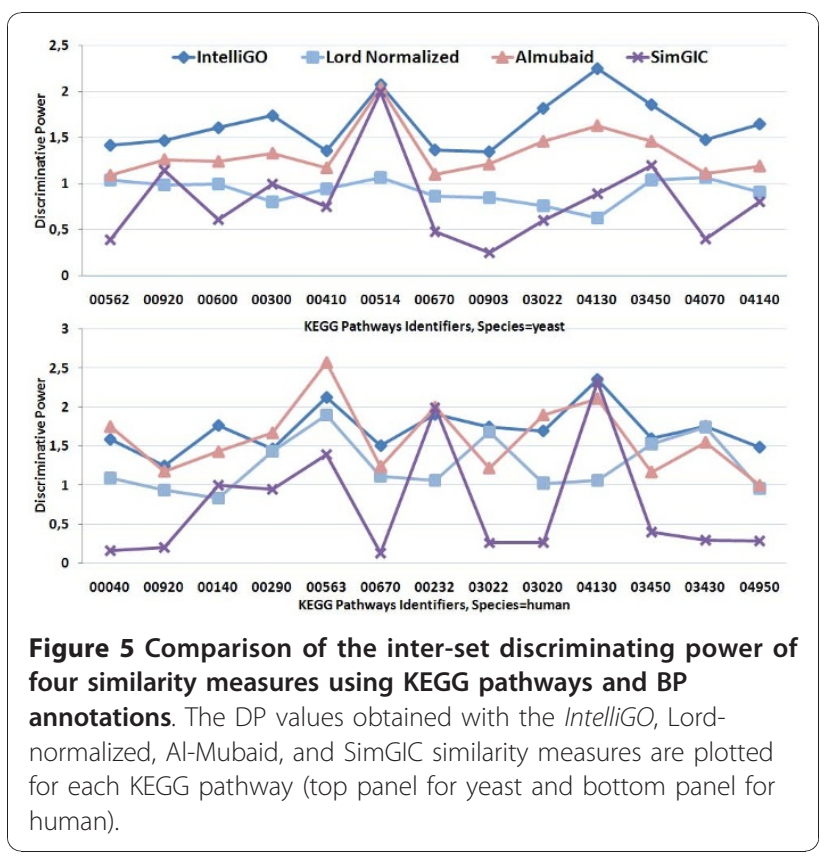

the $D P$ values obtained with the normalized Lord measure oscillate around 1.0 (especially for the yeast pathways), which is not desirable. The Al-Mubaid and SimGIC measures generate rather heterogeneous DP values ranging between 1.0 and 2.5 , and 0.2 and 2.3, respectively. Such heterogeneity indicates that the discriminative power of these measures is not as robust as the IntelliGO measure.

The results are very similar and even more favorable for the IntelliGO similarity measure when using Pfam clans and MF annotations as the benchmarking dataset

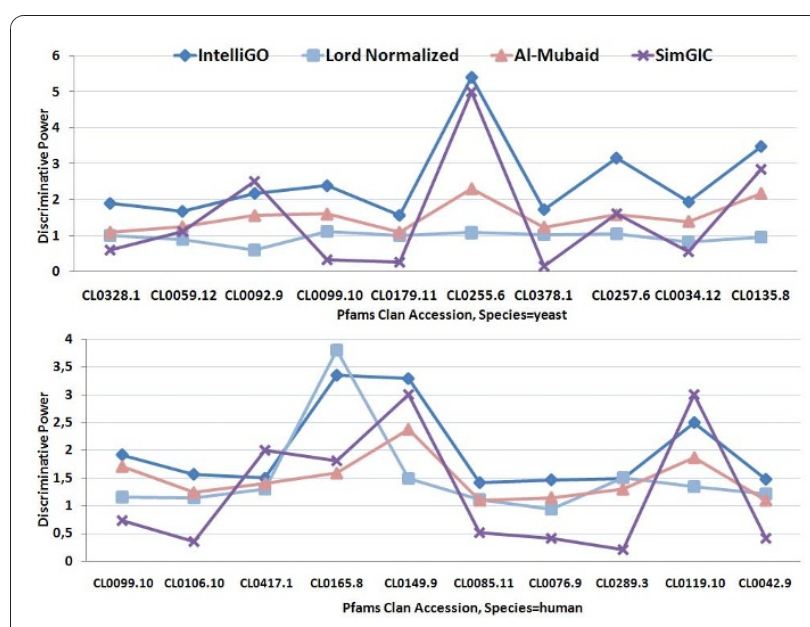

Figure 6 Comparison of the inter-set discriminating power of four similarity measures using Pfam clans and MF annotations. The DP values obtained with the IntelliGO, Lord-normalized, AlMubaid, and SimGIC similarity measures are plotted for each Pfam clan (yeast genes on top and human genes at bottom). 
(Figure 6). In this case, all of the IntelliGO DP values are greater than 1.5 , and give a maximum of 5.4 for Pfam clan CL0255.6. The three other measures give either very non discriminative values (e.g. Lord-normalized for yeast Pfam clans) or quite heterogeneous profiles (all other values). Overall, these results indicate that the IntelliGO similarity measure has a remarkable ability to discriminate between distinct sets of genes. This provides strong evidence that this measure will be useful in gene clustering experiments.

\subsubsection{Evaluation with the CESSM tool}

A complementary evaluation was performed using the recent Collaborative Evaluation of GO-based Semantic Similarity Measures (CESSM) tool. This on-line tool [51] enables the comparison of a given measure with previously published measures on the basis of their correlation with sequence, Pfam, and Enzyme Classification similarities [52]. It uses a dataset of 13,430 protein pairs involving 1,039 proteins from various species. These protein pairs are characterized by their sequence similarity value, their number of common Pfam domains and their degree of relatedness in the Enzyme Classification, leading to the so-called SeqSim, Pfam and ECC metrics. Semantic similarity values, calculated with various existing methods, are then analyzed against these three biological similarity indicators. The user is invited to upload the values calculated for the dataset with his own semantic similarity measure. The CESSM tool processes these values and returns the corresponding graphs, a table displaying the Pearson correlation coefficients calculated using the user's measure as well as 11 other reference measures along with calculated resolution values for each measure.

We present in Table 4 only the results obtained for correlation coefficients as they are the most useful for comparison purposes. The values obtained with the IntelliGO measure using the MF annotation and including or excluding GO terms with IEA evidence codes are shown in the last column. When the whole GO annotation is considered (first three lines), the correlation coefficients range from 0.40 for the SeqSim metrics to 0.65 for ECC metrics. The value obtained with the ECC metrics is higher than all other values reported for this comparison, the best being the SimUI measure (0.63). For the Pfam and SeqSim metrics, the correlation coefficients obtained with the IntelliGO measure are lower than the best values obtained from five and seven other measures, respectively, the best values being obtained from the SimGIC measure (0.63 and 0.71, respectively). When IEA annotations are excluded (the final three lines), the IntelliGO correlation coefficients are lower for the ECC and Pfam metrics, as observed with most other measures, but slightly higher for the SeqSim metrics. This limited increase or absence of decrease is observed with two other measures (SimUI, JA), whereas much larger increases are seen for the three Max variants of the Resnick, Lord, and Jaccard methods (RM, LM, JM). Hence, it appears that in this evaluation, the IntelliGO measure gives correlation values that are intermediate between those obtained with poor (RA, RM, LA, LM, JA, JM) and high (SimGIC, SimUI, RB, $\mathrm{LB}, \mathrm{JB})$ performance methods.

\section{Discussion}

Considering the growing number of semantic similarity measures, an important aspect of this study is the proposal of a method for estimating and comparing their performance. So far, rather heterogeneous and nonreproducible strategies have been used to validate new semantic similarity measures [9]. For example, it is generally assumed that gene products displaying sequence similarity should display similar MF annotations. This hypothesis was used by Lord et al. to evaluate their semantic similarity measure by exploring the correlation between gene annotation and sequence similarity in a set of human proteins [2]. They found a correlation between annotation and sequence similarity when using

Table 4 Evaluation results obtained with the CESSM evaluation tool

\begin{tabular}{|c|c|c|c|c|c|c|c|c|c|c|c|c|c|}
\hline \multicolumn{2}{|c|}{ Metrics } & \multicolumn{12}{|c|}{ Method } \\
\hline & & SimGIC & SimUI & RA & RM & RB & LA & LM & LB & JA & JM & $J B$ & IntelliGO \\
\hline & ECC & 0.62 & 0.63 & 0.39 & 0.45 & 0.60 & 0.42 & 0.45 & 0.64 & 0.34 & 0.36 & 0.56 & 0.65 \\
\hline & Pfam & 0.63 & 0.61 & 0.44 & 0.18 & 0.57 & 0.44 & 0.18 & 0.56 & 0.33 & 0.12 & 0.49 & 0.48 \\
\hline \multirow[t]{3}{*}{ All EC } & SeqSim & 0.71 & 0.59 & 0.50 & 0.12 & 0.66 & 0.46 & 0.12 & 0.60 & 0.29 & 0.10 & 0.54 & 0.40 \\
\hline & ECC & 0.58 & 0.57 & 0.37 & 0.47 & 0.48 & 0.38 & 0.51 & 0.51 & 0.37 & 0.46 & 0.51 & 0.48 \\
\hline & Pfam & 0.58 & 0.55 & 0.43 & 0.44 & 0.52 & 0.42 & 0.42 & 0.51 & 0.33 & 0.34 & 0.45 & 0.43 \\
\hline Non-IEA EC & SeqSim & 0.66 & 0.59 & 0.46 & 0.48 & 0.65 & 0.41 & 0.40 & 0.59 & 0.31 & 0.36 & 0.52 & 0.43 \\
\hline
\end{tabular}

Table 4: Pearson linear correlation coefficients are displayed for the ECC (Enzyme Classification Comparison), Pfam, and sequence similarity metrics (SeqSim). The Molecular Function GO annotation is used including (first three rows) or excluding (last three rows) annotation terms with IEA evidence codes. The column headings are listed as: SimUl: Union Intersection similarity; RA: Resnick Average; RM: Resnick Max; RB: Resnick Best match; LA: Lord Average; LM: Lord Max; LB: Lord Best match; JA: Jaccard Average; JM: Jaccard Max; JB: Jaccard Best match. 
the MF aspect of GO annotations, and this was later confirmed by Schlicker et al. [28] using a different similarity measure. These authors also tested their similarity measure for clustering protein families from the Pfam database on the basis of their MF annotations. They showed that Pfam families with the same function did form rather well-defined clusters. In this frame of mind, the CESSM tool used in this study (Section 2.4.5) is a valuable initiative towards standardizing the evaluation of semantic similarity measures.

Another group of evaluation techniques relies on the hypothesis that genes displaying similar expression profile should share similar functions or participate in similar biological processes. This was used by Chabalier et al. [41] to validate their similarity measure. These authors were able to reconstitute networks of genes presenting high pairwise similarity based on BP annotations and to characterize at least some of these networks with a particular transcriptional behavior and/or some matching with relevant KEGG pathways.

Using pathways as established sets of genes displaying functional similarity has also become an accepted way to validate new similarity measures. The analyses performed by Guo et al. [35] showed that all pairs of proteins within KEGG human regulatory pathways have significantly higher similarity than expected by chance in terms of BP annotations. Wang et al. [29] and AlMubaid et al. [26] have tested their similarity measure on yeast genes belonging to some pathways extracted from the Saccharomyces Genome Database. In the former study, only MF annotations were considered and the authors' similarity measure led to clustering genes with similar functions within a pathway much more efficiently than a measure based on Resnik's similarity between GO terms. In the latter study, the values obtained for pairwise gene similarity using BP annotations within each studied pathway were also more consistent than those obtained with a measure based on Resnik's similarity.

In our study, two benchmarking datasets of KEGG pathways and Pfam clans were used to test the performance of the IntelliGO similarity measure. Expressions for intra-set similarity and inter-set discriminating power were defined to carry out this evaluation. The testing hypotheses used here are that genes in the same pathway or Pfam clan should share similar BP or MF annotations, respectively. These datasets contain 465 and 218 genes, respectively, which is less then the CESSM evaluation dataset (1,039 proteins). However, the calculation of intra-set and inter-set similarities led to 67,933 pairwise comparisons which is larger than in the CESSM dataset (13,340 protein pairs).

\section{Conclusions and perspectives}

This paper presents IntelliGO, a new vector-based semantic similarity measure for the functional comparison of genes. The IntelliGO annotation vector space model differs from others in both the weighting scheme used and the relationships defined between base vectors. The definition of this novel vector space model allows heterogeneous properties expressing the semantics of $\mathrm{GO}$ annotations (namely annotation frequency of GO terms, origin of GO annotations through evidence codes, and term-term relationships in the GO graph) to be integrated in a common framework. Moreover, the IntelliGO measure avoids some inconveniences encountered with other similarity measures such as the problem of aggregating at best term-term similarities. It also solves rigorously the problem of multiple depth values for $\mathrm{GO}$ terms in the GO rDAG structure. Furthermore, the effect of annotation heterogeneity across species is reduced when comparing genes within a given species thanks to the use of IAF coefficients which are constrained to the given species. Our results show that the IntelliGO similarity measure is robust since it copes with the variability of gene annotations in each considered set of genes, thereby providing consistent results such as an intra-set similarity value of at least 0.50 and a discriminative power of at least 1.3. Moreover, it has been shown that the IntelliGO similarity measure can use ECs to estimate the relative contributions of GO annotations to gene functional similarity. In future work, we intend to use our similarity measure in clustering experiments using hierarchical and $K$ means clustering of our benchmarking datasets. We will also test co-clustering approaches to compare functional clustering using IntelliGO with differential expression profiles [24,53].

\section{Methods}

The $\mathrm{C}++$ programming language was used for developing all programs. The extraction of the LCAs and the SPLS of pairs of GO terms was performed by querying the GO relational database with the AmiGO tool [54].

\subsection{Reference Similarity Measures}

The four measures compared in our evaluation were implemented using the following definitions. Let $g_{1}$ and $g_{2}$ be two gene products represented by collections of GO terms $g_{1}=\left\{t_{1,1}, \ldots, t_{1, i}, \ldots, t_{1, n}\right\}$ and $g_{2}=\left\{t_{2,1}, \ldots, t_{2, i}, \ldots\right.$, $\left.t_{2, m}\right\}$. The first measure is Lord's similarity measure [2], 
which is based on Resnik's pairwise term similarity. For each pair of terms, $t_{i}$ and $t_{j}$, the Resnik measure is defined as the information content (IC) of their $L C A$ :

$$
\operatorname{SIM}_{\text {Resnik }}\left(t_{i}, t_{j}\right)=\operatorname{IC}\left(\operatorname{LCA}\left(t_{i}, t_{j}\right)\right)
$$

Then, the Lord similarity measure between $g_{1}$ and $g_{2}$ is calculated as the average of the Resnik similarity values obtained for all pairs of annotation terms:

$$
\operatorname{SIM}_{\text {Lord }}\left(g_{1}, g_{2}\right)=\frac{\sum_{i=1}^{n} \sum_{j=1}^{m} \operatorname{SIM}_{\text {Resnik }}\left(t_{1, i}, t_{2, j}\right)}{n * m} .
$$

Because this measure yields values greater than 1.0, we normalize the values obtained for a set of genes or for a collection of sets by dividing by the maximal value.

The second measure used here was introduced by AlMubaid et al. [31]. This method first calculates the shortest path length (PL) matrix between all pairs of GO terms annotating the two genes, i.e. PL $\left(t_{1, i}, t_{2, j}\right), \forall \mathrm{i}$ $\in[1, \mathrm{n}], \forall \mathrm{j} \in[1, \mathrm{~m}]$. It then calculates the average of all $\mathrm{PL}$ values in the matrix, which represents the average PL between the two gene products:

$$
\operatorname{PL}\left(g_{1}, g_{2}\right)=\frac{\sum_{i=1}^{n} \sum_{j=1}^{m} P L\left(t_{1, i}, t_{2, j}\right)}{n * m} .
$$

Finally, a transfer function is applied to this PL value to convert it into a similarity value. As this similarity monotonically decreases when the PL increases, the similarity value is obtained by:

$$
\operatorname{SIM}_{A l-M u b a i d}\left(g_{1}, g_{2}\right)=e^{-f^{*} P L\left(g_{1}, g_{2}\right)},
$$

with $f=0.2$ according to the authors.

The third measure used here is the classical weightedcosine measure, whereby each gene is represented by its annotation vector in an orthogonal VSM. Each component represents a GO term and is weighted by its own IAF value $\left(w_{i}=\operatorname{IAF}\left(t_{i}\right)\right)$ if the term annotates the gene, otherwise the weight is set to 0.0 . Then, the weightedcosine measure is defined in (20) but with the classical dot product expression in which $\vec{g}_{1} \cdot \vec{g}_{2}=\sum_{i} w_{i}^{2}$, for all terms $t_{i}$ present in both annotation vectors.

The last measure used here is SimGIC (Graph Information Content), which is also known as the Weighted Jaccard measure [36]. This measure is available in the csbl.go package within R Bioconductor [55], [56]. Given two gene products $g_{1}$ and $g_{2}$ represented by their two extended annotation sets (terms plus ancestors), the semantic similarity between these two gene products is calculated as the ratio between the sum of the information contents of GO terms in the intersection and the sum of the information contents of GO terms in the union:

$$
\operatorname{Sim} \operatorname{GIC}\left(g_{1}, g_{2}\right)=\frac{\sum_{t \in g_{1} \cap g_{2}} I C(t)}{\sum_{t \in g_{1} \cup g_{2}} I C(t)} .
$$

\subsection{Intra-Set and Inter-Set Similarity}

Consider $S$, a collection of sets of genes where $S=\left\{S_{1}\right.$, $\left.S_{2}, \ldots, S_{i}\right\}$ (a set $S_{k}$ can be a KEGG pathway or a Pfam clan). For each set $S_{k}$, let $\left\{g_{k 1}, g_{k 2}, \ldots . ., g_{k n}\right\}$ be the set of $n$ genes comprised in $S_{k}$. Let $\operatorname{Sim}(g, h)$ be a similarity measure between genes $g$ and $h$. The intra-set similarity value is defined for a given set of genes $S_{k}$ by:

$$
\operatorname{Intra\_ Set\_ Sim}\left(S_{k}\right)=\frac{\sum_{i=1}^{n} \sum_{j=1}^{n} \operatorname{Sim}\left(g_{k i}, g_{k j}\right)}{n^{2}} \text {. }
$$

For two sets of genes $S_{k}$ and $S_{l}$ composed of $n$ and $m$ genes respectively, we define the inter-set similarity value by:

$$
\text { Inter_Set_Sim }\left(S_{k}, S_{l}\right)=\frac{\sum_{i=1}^{n} \sum_{j=1}^{m} \operatorname{Sim}\left(g_{k i}, g_{l j}\right)}{n * m} .
$$

Note that when the Sim function takes values in the interval 0[1], so do the Intra_Set_Sim and Inter_Set_Sim functions. Finally, for a given collection $S$ composed of $p$ sets of genes, the discriminative power of the semantic similarity measure $\operatorname{Sim}$ with respect to a given set $S_{k}$ in $S$ will be defined as:

$$
D P_{S i m}\left(S_{k}\right)=\frac{(p-1) I n t r a \_S e t \_S i m\left(S_{k}\right)}{\sum_{i=1, i \neq k}^{p} I n t e r \_S e t \_S i m\left(S_{k}, S_{i}\right)} .
$$

\section{Acknowledgements}

This work was supported by the French National Institute of Cancer (INCa) and by the Region Lorraine program for research and technology (CPER MISN). SB is a recipient of an INCa doctoral fellowship. Special thanks to Dave Ritchie for careful reading of the manuscript.

\section{Author details}

'LORIA (CNRS, INRIA, Nancy-Université), Équipe Orpailleur, Bâtiment B, Campus scientifique, 54506 Vandoeuvre-lès-Nancy Cedex, France. ${ }^{2}$ L.B.G.I., CNRS UMR7104, IGBMC, 1 rue Laurent Fries, 67404 IIIkirch Strasbourg, France.

\section{Authors' contributions}

$\mathrm{SB}, \mathrm{MD}$ and $\mathrm{MS}$ designed the IntelliGO similarity measure. SB developed and implemented the method. MD and MS evaluated the biological results. MD, MS, OP and AN supervised the study, making significant contributions. SB, MD and MS wrote the paper. All authors proofread and approved the final manuscript. 
Received: 19 May 2010 Accepted: 1 December 2010

Published: 1 December 2010

\section{References}

1. Ashburner M, Ball C, Blake J, Botstein D, Butler H, Cherry M, Davis A, Dolinski K, Dwight S, Eppig J, Harris M, Hill D, Issel-Tarver L, Kasarskis A, Lewis S, Matese JC, Richardson J, Ringwald M, Rubin G, Sherlock G: Gene Ontology: tool for the unification of biology. Nature Genetics 2000, 25:25-29.

2. Lord PW, Stevens RD, Brass A, Goble CA: Investigating semantic similarity measures across the Gene Ontology: the relationship between sequence and annotation. Bioinformatics 2003, 19(10):1275-1283[http://bioinformatics. oxfordjournals.org/cgi/content/abstract/19/10/1275].

3. Consortium TGO: The Gene Ontology in 2010: extensions and refinements. Nucl Acids Res 2010, 38(suppl 1):D331-335.

4. Barrell D, Dimmer E, Huntley RP, Binns D, O'Donovan C, Apweiler R: The GOA database in 2009-an integrated Gene Ontology Annotation resource. Nucl Acids Res 2009, 37(suppl 1):D396-403.

5. Khatri $\mathrm{P}$, Draghici S: Ontological analysis of gene expression data: current tools, limitations, and open problems. Bioinformatics 2005, 21(18):3587-3595[http://bioinformatics.oxfordjournals.org/cgi/content/ abstract/21/18/3587]

6. Huang D, Sherman B, Tan Q, Collins J, Alvord WG, Roayaei J, Stephens R, Baseler M, Lane HC, Lempicki R: The DAVID Gene Functional Classification Tool: a novel biological module-centric algorithm to functionally analyze large gene lists. Genome Biology 2007, 8(9):R183[http://genomebiology. com/2007/8/9/R183].

7. Beissbarth T, Speed TP: GOstat: find statistically overrepresented Gene Ontologies within a group of genes. Bioinformatics 2004, 20(9):1464-1465 [http://bioinformatics.oxfordjournals.org/cgi/content/abstract/20/9/1464].

8. Speer N, Spieth C, Zell A: A Memetic Co-Clustering Algorithm for Gene Expression Profiles and Biological Annotation. 2004.

9. Pesquita C, Faria D, Falcão AO, Lord P, Couto FM: Semantic Similarity in Biomedical Ontologies. PLoS Comput Biol 2009, 5(7):e1000443.

10. Rogers MF, Ben-Hur A: The use of gene ontology evidence codes in preventing classifier assessment bias. Bioinformatics 2009, 25(9):1173-1177 [http://bioinformatics.oxfordjournals.org/cgi/content/abstract/25/9/1173].

11. The Gene Ontology Evidence Tree. [http://www.geneontology.org/GO. evidence.tree.shtml].

12. Du Z, Li L, Chen CF, Yu PS, Wang JZ: G-SESAME: web tools for GO-termbased gene similarity analysis and knowledge discovery. Nucl Acids Res 2009 [http://nar.oxfordjournals.org/cgi/content/abstract/gkp463v1], gkp463.

13. Popescu M, Keller JM, Mitchell JA: Fuzzy Measures on the Gene Ontology for Gene Product Similarity. IEEE/ACM Transactions on Computational Biology and Bioinformatics 2006, 3(3):263-274.

14. Ganesan P, Garcia-Molina H, Widom J: Exploiting hierarchical domain structure to compute similarity. ACM Trans Inf Syst 2003, 21:64-93.

15. Blanchard $E$, Harzallah M, Kuntz P: A generic framework for comparing semantic similarities on a subsumption hierarchy. 18th European Conference on Artificial Intelligence (ECAl) 2008, 20-24.

16. Tversky A: Features of similarity. Psychological Review 1977, 84:327-352.

17. Lee WN, Shah N, Sundlass K, Musen M: Comparison of Ontology-based Semantic-Similarity Measures. AMIA Annu Symp Proceedings 2008, V2008:384-388.

18. Resnik P: Using Information Content to Evaluate Semantic Similarity in a Taxonomy. IJCAl 1995, 448-453[http://citeseerx.ist.psu.edu/viewdoc/ summary?doi=10.1.1.55.5277].

19. Jiang JJ, Conrath DW: Semantic Similarity Based on Corpus Statistics and Lexical Taxonomy. International Conference Research on Computational Linguistics (ROCLING X) 1997, 9008+[http://www.bibsonomy.org/bibtex/ 2c4ffc507dafc908eab62fde53f7e4f7a/sdo].

20. Miller GA: WordNet: A Lexical Database for English. Communications of the ACM 1995, 38:39-41.

21. Wu Z, Palmer M: Verbs semantics and lexical selection. Proceedings of the 32nd annual meeting on Association for Computational Linguistics Morristown, NJ, USA: Association for Computational Linguistics; 1994, 133-138.

22. Lin D: An Information-Theoretic Definition of Similarity. ICML '98. Proceedings of the Fifteenth International Conference on Machine Learning San Francisco, CA, USA: Morgan Kaufmann Publishers Inc; 1998, 296-304.
23. Sevilla JL, Segura V, Podhorski A, Guruceaga E, Mato JM, Martinez-Cruz LA, Corrales FJ, Rubio A: Correlation between Gene Expression and GO Semantic Similarity. IEEE/ACM Trans. Comput. Biol. Bioinformatics 2005, 2(4):330-338.

24. Brameier M, Wiuf C: Co-Clustering and visualization of gene expression data and gene ontology terms for Saccharomyces cervisiae using self organizing maps. Biological Informatics 2007, , 40: 160-173.

25. Rada R, Mili H, Bicknell E, Blettner M: Development and application of a metric on semantic nets. Systems, Man and Cybernetics, IEEE Transactions on $1989,19: 17-30$

26. Nagar A, Al-Mubaid H: A New Path Length Measure Based on GO for Gene Similarity with Evaluation using SGD Pathways. Proceedings of the 2008 21st IEEE International Symposium on Computer-Based Medical Systems (CBMS 08) Washington, DC, USA: IEEE Computer Society; 2008, 590-595.

27. Floridi L: Outiline of a Theory of Strongly Semantic Information. Minds Mach 2004.

28. Schlicker A, Domingues F, Rahnenfuhrer J, Lengauer T: A new measure for functional similarity of gene products based on Gene Ontology. BMC Bioinformatics 2006, 7:302[http://www.biomedcentral.com/1471-2105/7/302].

29. Wang JZ, Du Z, Payattakool R, Yu PS, Chen CF: A new method to measure the semantic similarity of GO terms. Bioinformatics 2007, 23(10):1274-1281 [http://bioinformatics.oxfordjournals.org/cgi/content/abstract/23/10/1274].

30. Othman RM, Deris S, Illias RM: A genetic similarity algorithm for searching the Gene Ontology terms and annotating anonymous protein sequences. J of Biomedical Informatics 2008, 41:65-81.

31. Nagar A, Al-Mubaid H: Using path length measure for gene clustering based on similarity of annotation terms. Computers and Communications, 2008. ISCC 2008. IEEE Symposium on 2008, 637-642.

32. Martin D, Brun C, Remy E, Mouren P, Thieffry D, Jacq B: GOToolBox: functional analysis of gene datasets based on Gene Ontology. Genome Biology 2004, 5(12)[http://genomebiology.com/2004/5/12/R101].

33. Mistry M, Pavlidis P: Gene Ontology term overlap as a measure of gene functional similarity. BMC Bioinformatics 2008, 9:327 [http://www. biomedcentral.com/1471-2105/9/327].

34. The Bioconductor GOstats package. [http://bioconductor.org/packages/ 2.5/bioc/vignettes/GOstats/inst/doc/GOvis.pdf].

35. Guo X, Liu R, Shriver CD, Hu H, Liebman MN: Assessing semantic similarity measures for the characterization of human regulatory pathways. Bioinformatics 2006, 22(8):967-973 [http://bioinformatics.oxfordjournals.org/ cgi/content/abstract/22/8/967].

36. Pesquita C, Faria D, Bastos H, Ferreira A, Falcão AO, Couto F: Metrics for GO based protein semantic similarity: a systematic evaluation. $B M C$ Bioinformatics 2008, 9(Suppl 5):S4 [http://www.biomedcentral.com/14712105/9/S5/S4].

37. Salton G, McGill MJ: Introduction to Modern Information Retrieval McGrawHill; 1983.

38. Polettini N: The Vector Space Model in Information Retrieval-Term Weighting Problem. 2004

39. Bodenreider O, Aubry M, Burgun A: Non-lexical approaches to identifying associative relations in the gene ontology. The Gene Ontology. PBS 2005 2005, 91-102.

40. Glenisson P, Antal P, Mathys J, Moreau Y, Moor BD: Evaluation Of The Vector Space Representation In Text-Based Gene Clustering. Proc of the Eighth Ann Pac Symp Biocomp (PSB 2003) 2003, 391-402.

41. Chabalier J, Mosser J, Burgun A: A transversal approach to predict gene product networks from ontology-based similarity. BMC Bioinformatics 2007, 8:235 [http://www.biomedcentral.com/1471-2105/8/235].

42. Wright CC: The kappa statistic in reliability studies: use, interpretation, and sample size requirements. Physical Therapy 2005, 85(3):257-268.

43. Blott S, Camous F, Gurrin C, Jones GJF, Smeaton AF: On the use of Clustering and the MeSH Controlled Vocabulary to Improve MEDLINE Abstract Search. CORIA 2005, 41-56.

44. Couto FM, Silva MJ, Coutinho PM: Measuring semantic similarity between Gene Ontology terms. Data Knowl Eng 2007, 61:137-152.

45. The NCBI gene2go file. [ftp://ftp.ncbi.nih.gov/gene/DATA/gene2go.gz].

46. The AMIGO database. [http://amigo.geneontology.org].

47. The KEGG Pathways database. [http://www.genome.jp/kegg/pathway. html].

48. The DBGET database retrieval system. [http://www.genome.jp/dbget/].

49. The Sanger Pfam database. [http://pfam.sanger.ac.uk].

50. The Uniprot database. [http://www.uniprot.org/]. 
51. The Collaborative Evaluation of Semantic Similarity Measures tool. [http://xldb.di.fc.ul.pt/tools/cessm/].

52. Catia , Pessoa D, Faria D, Couto F: CESSM: Collaborative Evaluation of Semantic Similarity Measures. JB2009. Challenges in Bioinformatics 2009

53. Benabderrahmane S, Devignes MD, Smaïl Tabbone M, Poch O, Napoli A, Nguyen N-H N, Raffelsberger W: Analyse de données transcriptomiques: Modélisation floue de profils d'expression différentielle et analyse fonctionnelle. Actes du XXVIlième congrès Informatique des Organisations et Systèmes d'information et de décision - INFORSID 2009 Toulouse France: IRITToulouse; 2009, 413-428 [http://hal.inria.fr/inria-00394530/en/].

54. Carbon S, Ireland A, Mungall CJ, Shu S, Marshall B, Lewis S, the AmiGO Hub, the Web Presence Working Group: AmiGO: online access to ontology and annotation data. Bioinformatics 2009, 25(2):288-289[http://bioinformatics. oxfordjournals.org/cgi/content/abstract/25/2/288].

55. The csbl.go package. [http://csbi.ltdk.helsinki.fi/anduril/].

56. Ovaska K, Laakso M, Hautaniemi S: Fast Gene Ontology based clustering for microarray experiments. BioData Mining 2008, 1:11 [http://www. biodatamining.org/content/1/1/11].

57. The Pfam_C October 2009 release file. [ftp://ftp.sanger.ac.uk/pub/ databases/Pfam/releases/Pfam24.0/Pfam-C.gz].

doi:10.1186/1471-2105-11-588

Cite this article as: Benabderrahmane et al:: IntelliGO: a new vectorbased semantic similarity measure including annotation origin. $B M C$ Bioinformatics 2010 11:588.

\section{Submit your next manuscript to BioMed Central and take full advantage of:}

- Convenient online submission

- Thorough peer review

- No space constraints or color figure charges

- Immediate publication on acceptance

- Inclusion in PubMed, CAS, Scopus and Google Scholar

- Research which is freely available for redistribution

Submit your manuscript at www.biomedcentral.com/submit 